\title{
Grain and bedform roughness properties isolated from gravel-patch DEMs
}

\author{
Stephane Bertin ${ }^{1}$, Jane Groom ${ }^{1}$, and Heide Friedrich ${ }^{1, *}$ \\ ${ }^{1}$ Department of Civil and Environmental Engineering, University of Auckland, Auckland, New \\ Zealand
}

\begin{abstract}
Remote sensing of gravel-bed patches and resulting highresolution digital elevation models (DEMs) allow for the identification of various spatial scales of surface roughness. Thus far, dimensions relating to grain and bedform roughness scales have been determined using semivariograms or equivalent structure/autocorrelation functions. However, it is difficult to clearly differentiate roughness scales and separate analysis of roughness properties is not possible. This study examines the use of moving-window detrending on gravel-patch DEMs for isolating grain and bedform roughness and their respective topographic signatures. An extensive dataset of water-worked gravel surfaces collected in both laboratory and field environments is used. The measured bed topography is separated into two distinct DEMs: one representing grains, the other representing bedforms, and roughness properties are determined separately for grain and bedform DEMs. The results show that both roughness scales are controlled by the size of the coarse sediment forming the bed surface, with positive linear relationships connecting bed composition and vertical roughness. Coarse sediment is controlling bedform development by forming humps on the surface, in the lee of which finer sediment is sheltered. We present synthesis relationships connecting vertical roughness of gravel patches to the vertical roughness of grains and bedforms.
\end{abstract}

\section{Introduction}

High-resolution digital elevation models (DEMs) of water-worked gravel beds show the superposition of reliefs due to the arrangement of sediment grains onto low-amplitude bedforms [1,2].

Thus far, spatial scales of gravel-bed roughness have been examined using semivariograms or equivalent structure/autocorrelation functions [e.g., 3, 4], from which dimensions relating to grain and bedform roughness scales have been determined. Grain roughness, i.e., the roughness due to the ensemble of the grains, was referred to as the size and orientation of individual grains [4] while the arrangement of features several times the size of the largest grains on the riverbed was related to bedform (also called form or macro) roughness [3].

\footnotetext{
*Corresponding author: h.friedrich@auckland.ac.nz
} 
However, it is difficult to make a clear differentiation between roughness scales, as the analysis of semivariograms often requires a degree of judgment to associate observations of a transition between roughness scales (e.g., a break in slope) to the corresponding bed features $[4,5]$. Besides, separate analysis of grain and bedform roughness is not possible, so is developing relationships between roughness scales.

Despite recent progress in remote sensing, measuring gravel-bed microtopography in a range of practical situations remains challenging. This is because of challenging measurement environments and the need to resolve individual grain arrangement requiring high-resolution and accurate data. Even when measurement allows, application of highresolution topography in flow modelling studies is still hindered by the necessary trade-off between spatial extent and sampling resolution imposed by finite computing and time resources. It is however important to account for the small-scale riverbed topography in order to correctly characterise flow variability and turbulence [6]. Not knowing the relationships between roughness at the different scales for gravel beds, some authors have used data from grain size and general fractal properties of water-worked gravel surfaces for subgrid-scale roughness parameterization (i.e., the reconstruction of microtopography) [6]. The measurement of roughness parameters from the bed topography is another example for which it is important to explicitly account for the various spatial scales of roughness. For instance, the standard deviation of bed elevations $\left(\sigma_{\mathrm{Z}}\right)$, called vertical roughness, was initially calculated from DEMs after removal (i.e., detrending) of bed undulations larger than grain roughness. However, other studies calculated $\sigma_{\mathrm{Z}}$ from the bed topography without prior detrending of bedform roughness [7]. This makes comparing bed roughness between studies difficult, as the quantities measured and subsequently compared do not necessarily encompass the same scales of surface roughness.

In this paper, we examine relationships between low-amplitude bed undulations developed by water working (which we call bedforms) and the relief created by sediment grains' arrangement. In an attempt to provide general findings applicable for both controlled and uncontrolled gravel-bed environments, a large dataset, consisting of 35 water-worked gravel-bed patches from both the laboratory (17 DEMs) and the field (18 DEMs), is used for the analysis. Moving-window detrending [e.g., 2, 12] was applied to each of the 35 DEMs to separate bed undulations from the topography due to the grains, leading to two distinct surfaces: one representing grains (which we call the "grain DEMs"), the other representing small bedforms (called "the bedform DEMs"). The novelty of the study is the separate examination of grain and bedform roughness isolated from DEMs, allowing to determine roughness signatures for both grain and bedform DEMs and to examine inter-relationships between grain and bedform roughness and their formative controls. A more comprehensive study is published [8], where one can find detailed discussions of gravel-bed roughness parameterization and practical applications.

\section{Methods}

\subsection{Gravel-bed patches and the data examined}

The data examined comprise 21 gravel patches measured by the authors and 14 gravel patches collected by others and already presented elsewhere [7,9]. The data and more detailed analysis and results are presented in [8]. All DEMs satisfied the precondition of highresolution data necessary for grain roughness analysis, as evidenced by a small DEM grid spacing $(<4 \mathrm{~mm})$ compared to the size of the bed-surface material. Characteristic grain sizes of the bed surface (e.g., $\mathrm{D}_{50 \mathrm{~A}}$, the grain size for which $50 \%$ of the bed surface material is 
smaller) were all determined using Fehr's line-by-number method, facilitating comparisons between datasets (Fig. 1).

Data collection by the authors was made with digital photogrammetry [10] using a pair of Nikon D5100 cameras (16.4 Mpixel, $23.6 \times 15.6 \mathrm{~mm}^{2}$ sensor size), with Nikkor $20 \mathrm{~mm}$ lenses. This allowed the collection of raster DEMs with a final grid spacing equal to $1 \mathrm{~mm}$. 4 DEMs were collected in a laboratory flume for a study on stable fluvial armours [11]. The authors also measured 17 gravel patches on exposed gravel bars in the Whakatiwai River, a small gravel-bed stream located in New Zealand North Island. DEMs were characterised by a vertical accuracy of $\sim 0.4 \mathrm{~mm}$ and $\sim 0.7 \mathrm{~mm}$ in the laboratory and the field, respectively, while DEM size varied between $0.5 \times 0.5 \mathrm{~m}^{2}$ and $1.851 \times 0.378 \mathrm{~m}^{2}$.

Stable armours formed in a laboratory flume and presented in [7] represent 13 of the gravel patches analysed hereafter. A laser displacement meter was used for DEM measurement (downstream and transverse point spacing of $1 \mathrm{~mm}$ and $4 \mathrm{~mm}$, respectively) over an area of $2.4 \times 0.716 \mathrm{~m}^{2}$ with a stated vertical accuracy of $0.1 \mathrm{~mm}$.

Another field DEM used in the study was collected from the Waimakariri River in New Zealand South Island [9], using a hand-held laser scanner swept across the exposed gravel patch $\left(0.89 \times 0.618 \mathrm{~m}^{2}\right)$. The DEM provided to the authors had a grid spacing of $2 \mathrm{~mm}$ with a stated vertical accuracy of $1 \mathrm{~mm}$.

Fig. 1A presents the $\mathrm{D}_{50 \mathrm{~A}}$ and $\mathrm{D}_{84 \mathrm{~A}}$ for all the gravel patches examined in this study. The latter differ largely in their surface sediment size, as shown by $\mathrm{D}_{84 \mathrm{~A}}$ varying between $23 \mathrm{~mm}$, for a patch collected in the laboratory, to nearly $105 \mathrm{~mm}$ for a gravel patch measured in the field. Likewise, Fig. 1B shows that the gravel beds formed in a laboratory flume differed in their formative flow conditions, with the Shields stress $\tau^{*}$ covering the range 0.04 to 0.11 . The dataset available for the study is therefore representative of a variety of gravel riverbed conditions, and it is expected that the analysis presented below will provide general results applicable to a wide range of data.

\subsection{Isolating roughness scales from DEMs}

Planar trends originating from bed/flume slope and from the orientation of the measurement device with respect to the bed surface were first removed from the DEMs using a leastsquares fit procedure. The DEMs after linear detrending are hereafter referred to as the "measured DEMs". Secondly, moving-window detrending (see Fig. 2) was applied to the measured DEMs to isolate any bed undulations (e.g., humps and hollows on the surface) larger than the biggest individual particles (cf. the red line presented in Fig. 3). Following the method presented in [12], a trend surface was fitted to a regular grid with point spacing equal to $1.25 \mathrm{D}_{90 \mathrm{~A}}$, and the elevations of grid points were determined by averaging measured elevations within a circle with a diameter of $2.5 \mathrm{D}_{90 \mathrm{~A}}$ centred on the grid point. Bicubic spline interpolation is used to interpolate between trend surface grid points at the location of the measured DEM points. The interpolated surfaces represent the "bedform DEMs", and subtracting these surfaces from the measured DEMs isolates the topography due to the sediment grains, hence produces the "grain DEMs".

\subsection{Data analysis}

The same analysis was performed for all measured, grain and bedform DEMs, in an attempt to characterise differences between grain and bedform roughness, and to illustrate eventual relationships. The analysis presented in this paper focuses on the standard deviation of bed elevations, $\sigma_{Z}$, which is a robust measure of vertical roughness. In [8], the analysis is extended to other roughness parameters, such as the length of correlations in bed elevations determined 
from second-order structure functions of bed elevations and to the inclination index representing grain imbrication.
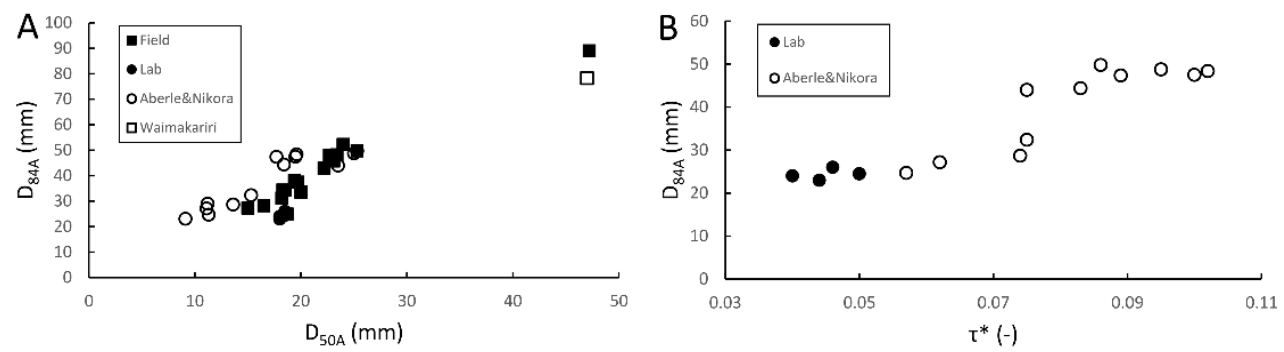

Fig. 1. Scatter plots presenting the range of surface sediment size for the gravel patches examined in this study (A), and the relationship between surface sediment size $\left(\mathrm{D}_{84 \mathrm{~A}}\right)$ and the Shields stress $\tau^{*}$, calculated as $\mathrm{u}^{* 2} /(\mathrm{SG}-1) \mathrm{gD}_{50}$ (where $\mathrm{u}^{*}$ is shear velocity, $\mathrm{SG}$ and $\mathrm{D}_{50}$ are specific gravity and median sediment size for the bulk sediment), for the gravel patches formed in a laboratory flume for which flow conditions are known (B).
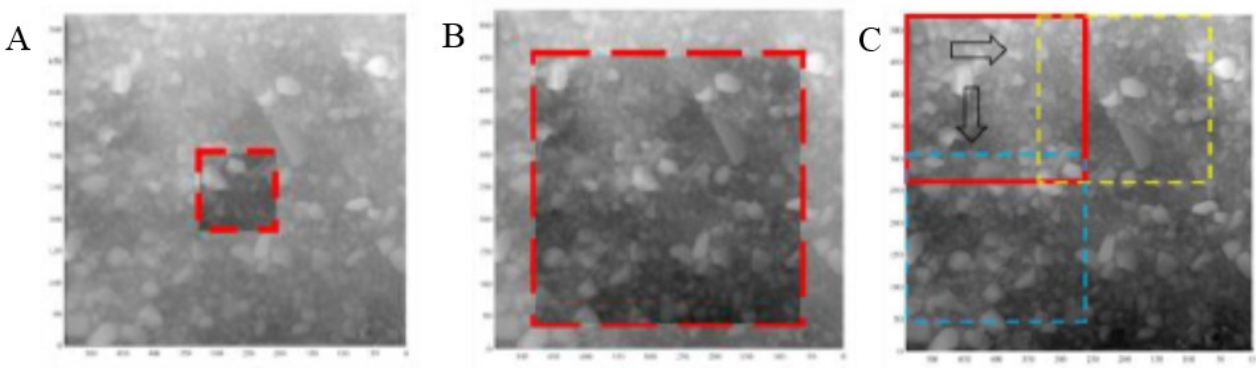

Fig. 2. Example of moving-window size selection based on $\mathrm{D}_{50 \mathrm{~A}}$ values. (A) $4 \times$ D $50 \mathrm{~A}$ or (B) $16 \times$ D50A in both directions. (C) Moving-window analysis with user defined window size (e.g. $10 \times$ D $50 \mathrm{~A}$ in both directions), with $25 \%$ overlap for clarity. Black arrows indicate moving window in both directions. New windows are defined with coloured dashed lines.

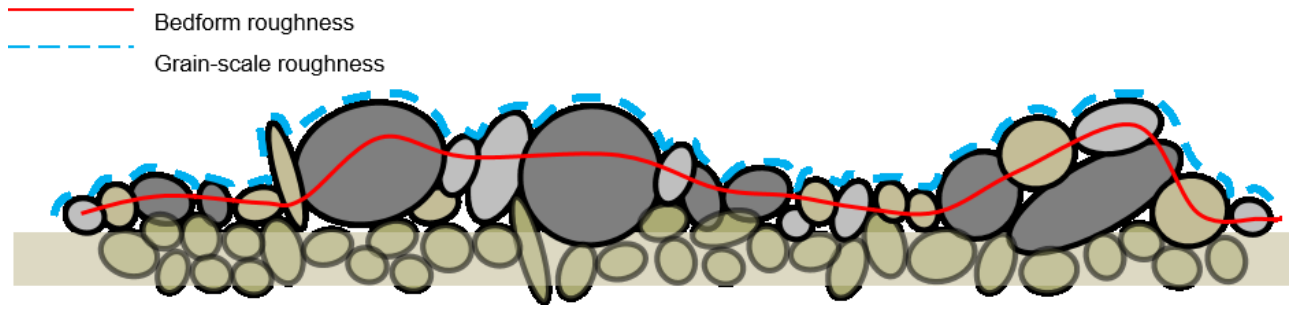

Fig. 3. Schematic presenting the superposition of the grain topography over bed undulations (i.e., bedforms) formed with water-working (from [13]).

A moving-window analysis technique (not to be confused with moving-window detrending used for isolating roughness scales) was used to quantify the spatial variations in roughness parameters within DEMs. In this case, roughness parameters were calculated over moving square windows of size proportional to the surface material $D_{50 \mathrm{~A}}$. A $95 \%$ overlap between moving windows enabled robust quantification of spatial variability, even at large window sizes. 


\section{Results and discussion}

A patch collected by the authors in the field serves as an example to test the ability of movingwindow detrending to separate grain and bedform roughness scales in this paper (Fig. 4). The patch show the superposition of grain topography (Fig. 4A) and low-amplitude bed undulations (Fig. 4B). The topographies representing grains and bedforms were correctly isolated using moving-window detrending. As a result, grain DEMs have a flat mean-bed level with high-frequency irregularities due to the grains (Fig. 4A), while bedform DEMs show smooth topographies, which represent the lower-frequency bed undulations (Fig. 4B).

Visually, bed undulations appear to be dependent on the arrangement of coarse sediment, with humps in the surface generally associated with groupings of coarse particles into smallscale structures such as clusters, lines, and stone cells, whereas smaller particles are confined in shelters formed by hollows. This assertion, which is supported in consideration of bedform stability, will be examined for all DEMs.

The spatial extent of grain and bedform roughness scales was examined using the movingwindow analysis technique for 4 DEMs, allowing the identification of consistent patterns. Vertical roughness $\left(\sigma_{\mathrm{Z}}\right)$ varies across a gravel patch (i.e., large variability and unstable median values at small window sizes) and that a sufficient window (hence DEM) size is necessary to obtain stable measures of $\sigma_{Z}$ representative of the surface. Stable measures of vertical grain roughness (i.e., $\left.\sigma_{Z, G}\right)$ are attained for square window sizes above $\sim\left(12-18 \mathrm{D}_{50 \mathrm{~A}}\right)^{2}$. Larger DEMs are necessary to determine stable measures of bedform roughness representative of the surface. $\sigma_{Z}$ of the measured DEMs is different from the sum of $\sigma_{Z}$ obtained separately from the grain DEMs and the bedform DEMs (i.e., $\sigma_{Z} \neq \sigma_{Z, G}+\sigma_{Z, B}$ ), which statistically, indicates a correlation between $\sigma_{\mathrm{Z}, \mathrm{G}}$ and $\sigma_{\mathrm{Z}, \mathrm{B}}$. Although previous work derived roughness from flow resistance models, not from the bed topography, it already observed the inadequacy of summing individual roughness components to derive the total roughness of the bed, which was explained by interaction effects between roughness scales [14].
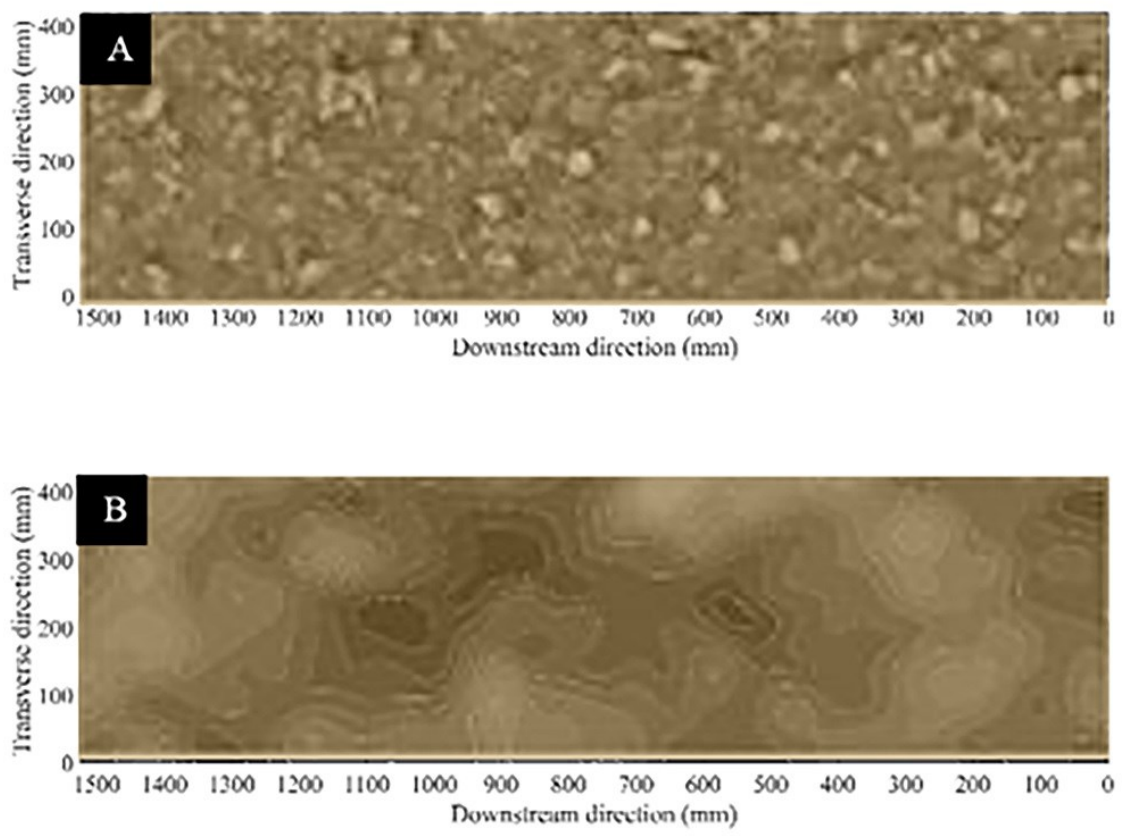

Fig. 4. Example of DEMs following moving-window detrending, the grain DEM (A) shows the topography due to the grains alone, while the bedform DEM (B) shows the underlying bed undulations. 
The relationships between vertical roughness $\left(\sigma_{\mathrm{z}}\right)$ and characteristic grain sizes of the bedsurface material are stronger as percentiles of the bed-surface grain-size distribution increase (Fig. 5). This suggests that for the patches examined, the size of the coarse surface sediment is a better indicator of the patch topography represented by $\sigma_{\mathrm{Z}}$. Besides, relationships are stronger when sediment size is compared to $\sigma_{\mathrm{Z}, \mathrm{G}}$ for the three percentiles. The strongest relationship $\left(\mathrm{R}^{2}=0.92, \mathrm{p}<0.01\right)$ is between $\sigma_{\mathrm{Z}, \mathrm{G}}$ and $\mathrm{D}_{84 \mathrm{~A}}($ Fig. $5 \mathrm{E})$. A difference in surface sediment size between patches is therefore a good explanation of the different grain topographies. Differences in bedform topography between patches (i.e. $\sigma_{Z, B}$ ) are not explained as well by the models (Fig. 5C, 5F and 5I).

The relationships developed in Fig. 5 were directly helpful to test the hypothesis that coarser sediment controls bedform geometry. For all 35 gravel patches, the bedform DEMs were used to delineate regions pertaining to humps or hollows on the surface, corresponding to bedform elevations above $1 \mathrm{~mm}$ and below $-1 \mathrm{~mm}$, respectively. As a proxy of sediment size, $\sigma_{\mathrm{Z}, \mathrm{G}}$ was measured for humps and hollows separately, and a relationship presented in Fig. $5 \mathrm{E}\left(\sigma_{\mathrm{Z}, \mathrm{G}}=0.17 \mathrm{D}_{84 \mathrm{~A}}-1.31, \mathrm{R}^{2}=0.92\right)$ provided estimates of $\mathrm{D}_{84 \mathrm{~A}}$. It confirms that sediment is coarser on bedform tops (i.e., humps) and finer in the troughs (i.e., hollows). Separate analysis of the laboratory data for which formative flow conditions are known indicates that the ratio of $D_{84 \mathrm{~A}}$ between humps and hollows $\left(\mathrm{D}_{\mathrm{hu}} / \mathrm{D}_{\mathrm{ho}}\right)$ increases significantly with both formative discharge and Shields stress increases $\left(\mathrm{R}^{2}=0.91\right.$ for both relations, $\mathrm{p}<$ 0.01 ), suggesting a better horizontal sorting of the bed-surface material at high formative discharge.
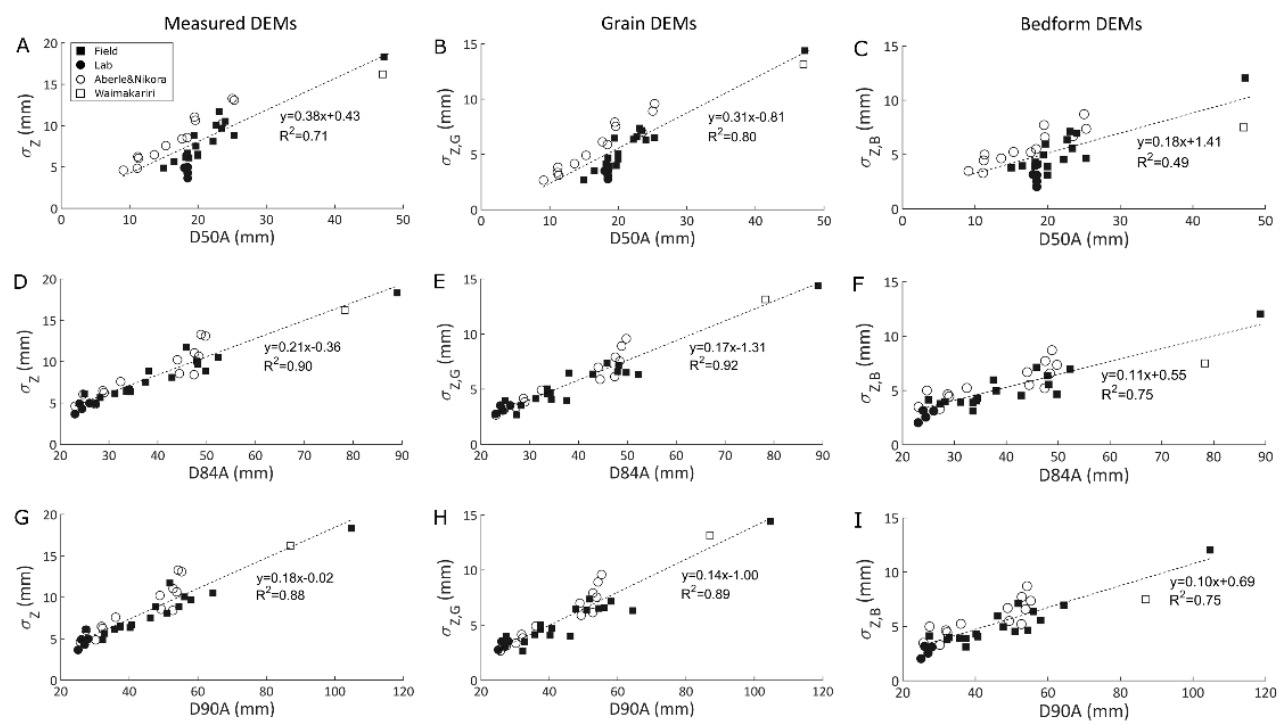

Fig. 5. Relationships between the standard deviation of bed elevations ( $\sigma \mathrm{Z}, \sigma \mathrm{Z}, \mathrm{G}$, and $\sigma \mathrm{Z}, \mathrm{B})$, representing vertical roughness, determined from $(\mathrm{A}, \mathrm{D}, \mathrm{G})$ the measured DEMs, $(\mathrm{B}, \mathrm{E}, \mathrm{H})$ the grain DEMs, and $(\mathrm{C}$, $\mathrm{F}, \mathrm{I})$ the bedform DEMs, respectively, and characteristic grain sizes (D50A, D84A and D90A) of the bedsurface material. Functional lines (i.e., corrected least-squares lines) best representing the data are based on the 35 DEMs available. Closed symbols represent data collected by the authors, while open symbols represent data collected by others and made available to the authors. Round symbols are the laboratory surfaces; square symbols the field surfaces (from [8]).

We also measured evolving gravel-bed topography collectively with the topography due to grain arrangements and the underlying bed undulations. $\sigma_{\mathrm{Z}}$ is strongly correlated to both $\sigma_{\mathrm{Z}, \mathrm{G}}$ measured from the grain DEMs $\left(\mathrm{R}^{2}=0.96\right)$ and $\sigma_{Z, B}$ measured from the bedform DEMs $\left(\mathrm{R}^{2}\right.$ $=0.89)$. However, the two roughness scales have different relation coefficients $(\mathrm{p}<0.05)$ 
when it comes to their imprint on the measured patch topography. Across most gravel patches in this study we observed that bedform roughness has a lesser impact on measured $\sigma_{Z}$ (i.e. $\sigma_{Z, B}$ is smaller than $\left.\sigma_{Z, G}\right)$. Assuming equal weight is given to the grain and the bedform function, a general relationship of the form $\sigma_{\mathrm{Z}}=0.71 \sigma_{\mathrm{Z}, \mathrm{G}}+0.82 \sigma_{\mathrm{Z}, \mathrm{B}}$ (mean residual error $=$ $0.31 \mathrm{~mm}$ ) represents gravel-bed vertical roughness embodied in patch-scale DEMs available for this study.

\section{Conclusion}

Remote sensing of water-worked gravel beds now allows the collection of high-resolution DEMs, from which the surface roughness can be determined. Previous work relying on semivariograms has shown that gravel-bed roughness is scale dependent. However, relationships between roughness scales have not be obtained.

This study has shown the possibility of using a moving-window detrending technique to isolate grain and bedform roughness from patch-scale gravel-bed DEMs. Resulting from roughness scale separation, roughness signatures of grains and bedforms can be studied separately. The analysis was applied to an extensive dataset comprising 35 water-worked gravel surfaces of varying size and resolution, collected in either a laboratory flume or the field.

The results demonstrate that measures of grain roughness are easier to determine at smaller DEM sizes than bedform roughness signatures, which requires larger DEM sizes. Grain vertical roughness is associated with the size of the coarse sediment forming the bed surface. Coarse sediment also controls bed undulations (bedforms) by forming humps on the surface, in the lee of which finer sediment is sheltered. Finally, we showed that patch-scale vertical roughness represented by $\sigma_{Z}$ can be estimated by knowing either grain vertical roughness or bedform vertical roughness (relationships presented in Fig. 5). This is an important finding for two reasons. Firstly, it provides an opportunity for flow modellers to parameterize gravel-bed microtopography from the smoothed beds used as input surfaces, helping to obtain realistic findings on flow variability and turbulence. Secondly, it provides new means to compare measures of surface roughness (here $\sigma_{z}$ ) found in the literature, which were obtained using different detrending techniques. Important work remains to be done linking bed topography and flow properties in gravel-bed rivers. The combined use of topography and flow remote sensing may ultimately allow realistic characterisation of the topographic controls on flow resistance.

\section{Acknowledgements}

The analysis was partly undertaken using a High-Performance Computing (HPC) platform provided by the New Zealand eScience Infrastructure (NeSI). The study was supported by the Marsden Fund (grant UOA1412) administered by the Royal Society of New Zealand.

\section{References}

1. G. Pender, T. B. Hoey, C. Fuller, I. K. McEwan, J. Hydraul. Res. 39, 3, 269-277 (2001)

2. D. M. Powell, A. Ockelford, S. P. Rice, J. K. Hillier, T. Nguyen, I. Reid, N. J. Tate, D. Ackerley, J. Geophys. Res. Earth Surf., 1494-1515 (2016).

3. A. Robert, Math. Geol., 20, 3, 205-225 (1988).

4. J. B. Butler, S. N. Lane, J. H. Chandler, Math. Geol., 33, 3, 301-330 (2001).

5. N. Clifford, A. Robert K. Richards, ESPL, 17, 2, 111-126 (1992).

6. M. A. Casas, S. N. Lane, R. J. Hardy, G. Benito, P. J., Water Resour. Res., 46, 3 (2010).

7. J. Aberle, V. Nikora, Water Resour. Res., 42, 11, W11414 (2006). 
8. S. Bertin, S., J. Groom, H. Friedrich, Water Resour. Res., 53 (2017).

9. G. Smart, J. Aberle, M. Duncan, J. Walsh, J. Hydraul. Res., 42, 3, 227-237 (2004).

10. S. Bertin, H. Friedrich, P. Delmas, E. Chan, G. Gimel'farb, ISPRS J. Photogramm. Remote Sens., 101, 0, 193-208 (2015).

11. S. Bertin, and H. Friedrich, Geomorphology, 306, 64-79 (2018).

12. G. Smart, M. Duncan, J. Walsh, J. Hydraul. Eng., 128, 6, 568-578 (2002).

13. J. Groom, S. Bertin, H. Friedrich. Proc 37th IAHR World Congress, 655-664 (2017).

14. R. D. Hey, Journal of Hydraulic Engineering, 114, 12, 1498-1508 (1988). 